\title{
Hepatic Induction of Fatty Acid Binding Protein 4 Plays a Pathogenic Role in Sepsis in Mice
}

\author{
Bingfang Hu, ${ }^{* \dagger}$ Yujin Li, ${ }^{*} \mathrm{Li} \mathrm{Gao,}{ }^{\dagger \dagger}$ Yan Guo, ${ }^{\dagger \S}$ Yiwen Zhang, ${ }^{*}$ Xiaojuan Chai, ${ }^{\dagger \uparrow}$ Meishu Xu, ${ }^{\dagger}$ Jiong Yan, ${ }^{\dagger}$ Peipei Lu,
} Songrong Ren, ${ }^{\dagger}$ Su Zeng, ${ }^{\circledR}$ Yulan Liu, ${ }^{\dagger \dagger}$ Wen Xie, ${ }^{\dagger \mid \|}$ and Min Huang*

\begin{abstract}
From the Institute of Clinical Pharmacology and Guangdong Provincial Key Laboratory of New Drug Design and Evaluation,* Sun Yat-Sen University, Guangzhou, China; the Department of Pharmaceutical Sciences, ${ }^{\dagger}$ Center for Pharmacogenetics, and the Department of Pharmacology and Chemical Biology," University of Pittsburgh, Pittsburgh, Pennsylvania; the Department of Gastroenterology, ${ }^{\ddagger}$ Peking University People’s Hospital, Beijing, China; the Department of Pathology, ${ }^{\S}$ Ruijin Hospital, Shanghai Jiao Tong University School of Medicine, Shanghai, China; and the Department of Pharmaceutical Analysis and Drug Metabolism, "Zhejiang Province Key Laboratory of Anti-Cancer Drug Research, College of Pharmaceutical Sciences, Zhejiang University, Hangzhou, China
\end{abstract}

\author{
Accepted for publication \\ January 5, 2017. \\ Address correspondence to \\ Min Huang, Ph.D., Institute \\ of Clinical Pharmacology, Sun \\ Yat-Sen University, Guangz- \\ hou 510080, China; or Wen \\ Xie, M.D., Ph.D., Center for \\ Pharmacogenetics, University \\ of Pittsburgh, Pittsburgh, PA \\ 15261. E-mail: huangmin@ \\ mail.sysu.edu.cn or wex6@pitt. \\ edu.
}

\begin{abstract}
Sepsis is defined as the host's deleterious systemic inflammatory response to microbial infections. Herein, we report an essential role of the fatty acid binding protein 4 (FABP4; alias adipocyte protein 2 or aP2), a lipid-binding chaperone, in sepsis response. Bioinformatic analysis of the Gene Expression Omnibus data sets showed the level of FABP4 was higher in the nonsurvival sepsis patients' whole blood compared to the survival cohorts. The expression of Fabp4 was induced in a liver-specific manner in cecal ligation and puncture (CLP) and lipopolysaccharide treatment models of sepsis. The induction of Fabp4 may have played a pathogenic role, because ectopic expression of Fabp4 in the liver sensitized mice to CLP-induced inflammatory response and worsened the animal's survival. In contrast, pharmacological inhibition of Fabp4 markedly alleviated the CLP responsive inflammation and tissue damage and improved survival. We conclude that FABP4 is an important mediator of the sepsis response. Early intervention by pharmacological inhibition of FABP4 may help to manage sepsis in the clinic. (Am J Pathol 2017, 187: 1059-1067; http://dx.doi.org/10.1016/j.ajpath.2017.01.002)
\end{abstract}

Sepsis is defined as the host's deleterious systemic inflammatory response to microbial infections. Because of its high incidence and associated mortality, sepsis has become an important life-threatening noncardiovascular disease in intensive care units. ${ }^{1}$ Previous studies suggest that many inflammatory factors play roles in the pathogenesis of sepsis. ${ }^{2}$ Two commonly used mouse models of sepsis are the cecal ligation and puncture (CLP) and treatment with lipopolysaccharide (LPS). ${ }^{3,4}$ Both models result in marked inflammatory response in vivo.

Fatty acid binding protein 4 (FABP4), alias adipocyte protein 2, is a lipid-binding chaperone most abundantly expressed in the adipocytes and macrophages. ${ }^{5,6}$ Previous reports suggested that FABP4 can aggravate the severity of inflammatory-related disease by increasing the levels of chemokines, such as the tumor necrosis factor- $\alpha$ (TNF- $\alpha)^{7}{ }^{7}$ Genetic deletion of FABP4 in the adipose tissue attenuated type II diabetes-associated insulin resistance. ${ }^{8}$ Moreover, pharmacological inhibition of FABP4 alleviated LPStriggered nonalcoholic steatohepatitis and ischemia/ reperfusion-induced liver injury. 9,10 Mechanistic studies revealed that FABP4 is a transcriptional target of several transcriptional factors, such as the peroxisome proliferatoractivated receptor- $\gamma^{11}$ and hypoxia-inducible factor $1 \alpha .^{10}$

Compared to adipose tissue and macrophages, the role of FABP4 in hepatocytes and the liver is relatively unknown. The basal expression of FABP4 in the liver is low, but the expression is highly inducible in response to

\footnotetext{
Supported in part by NIH grant ES023438 (W.X.), Natural Science Foundation of China grant 81320108027 (M.H.), and the Chinese Ministry of Education 111 Project grant B16047 (M.H.). B.H. was supported by a scholarship from Government of China's China Scholarship Council (201206380045)

B.H., Y.L., and L.G. contributed equally to this work.

Disclosures: None declared.
} 
ischemia/reperfusion injury. ${ }^{10}$ Sepsis is a systemic inflammatory disease that injures the liver. ${ }^{10}$ It is unknown whether FABP4 plays a role in the pathogenesis of sepsis and if so, whether the pathogenic effect is mediated by the hepatic FABP4.

In this study, we found the level of FABP4 was negatively associated with the survival of septic patients. The expression of Fabp4 was induced in mouse models of sepsis. Moreover, pharmacological inhibition of Fabp4 protected mice from sepsis, whereas ectopic expression of Fabp4 in the liver sensitized mice to CLP-induced lethality.

\section{Materials and Methods}

\section{Bioinformatic Analysis of the Gene Expression Omnibus Data Sets}

The mRNA expression of FABP4 in septic patients was analyzed in GSE54514 (whole blood transcriptome) and GSE13205 (skeletal muscle transcriptome) data sets from the Gene Expression Omnibus database (http://www.ncbi. nlm.nih.gov/geo; accession numbers GSE54514 and GSE13205, respectively).

\section{Animals and Drug Treatment}

C57BL/6 mice were purchased from the Jackson Laboratory (Bar Harbor, ME). All animal experiments were performed with female mice of 8 to 10 weeks of age. When necessary, mice were pretreated with a daily gavage of vehicle (saline) or $30 \mathrm{mg} / \mathrm{kg}$ BMS309403 for 5 consecutive days before the initiation of sepsis models. In the survival experiments, the BMS309403 treatment continued until the end time points. When necessary, a single tail vein injection of adenovirus encoding the control luciferase (Ad-Luc) or mouse FABP4 (Ad-Fabp4) was performed 7 days before being challenged with CLP. The Ad-Fabp4 virus was a gift from Dr. Karen Lam (University of Hong Kong, Hong Kong). To deplete the Kupffer cells, mice were treated with saline or $20 \mathrm{mg} / \mathrm{kg}$ gadolinium chloride via tail vein injection. Twenty-four hours later, the second injection of gadolinium chloride was given immediately before the i.p. injection of LPS. The FABP4 inhibitor BMS309403 was purchased from Chemrenblock Technology (Jiangsu, China). All other chemicals were purchased from Sigma (St. Louis, MO). The use of mice in this study was approved by the University of Pittsburgh's Institutional Animal Care and Use Committee.

\section{The LPS and CLP Models of Sepsis}

The models were essentially used as previously described. ${ }^{12}$ In the LPS model, LPS was dissolved in phosphate-buffered saline and given by i.p. injection at the dose of $5 \mathrm{mg} / \mathrm{kg}$. In the CLP model, mice were anesthetized with a mixture of $150 \mathrm{mg} / \mathrm{kg}$ ketamine and $10 \mathrm{mg} / \mathrm{kg}$ xylazine. Under aseptic conditions, a 2-cm midline laparotomy was performed to expose the cecum. The cecum was then ligated in $50 \%$ with a 4.0 silk suture and was perforated twice with a needle. ${ }^{13,14}$ The 18- and 21-gauge needles were used in the BMS309403 model and the Ad-Fabp4 model, respectively. The cecum was then gently squeezed to extrude a few feces from the perforation sites. The cecum was then returned to the peritoneal cavity and the laparotomy was closed with 4.0 silk sutures. Mice in the sham group received the 2-cm midline laparotomy without the cecum procedure. Mice were returned to their home cages after they recovered from the surgical anesthetization. The mice were sacrificed by carbon dioxide euthanasia and the liver, gonadal (female)/ epididymal (male) white adipose tissue, lung, and blood samples were collected 12 hours after the surgery. For the survival experiments, mice were subjected to CLP and were monitored for mortality over a 7-day period.

\section{Real-Time PCR and Western Blot Analysis}

Total RNA was extracted by using the TRIzol reagent from Thermo Fisher Scientific Inc. (Carlsbad, CA) or RNAprep pure Hi-blood kit from TIANGEN BIOTECH (Beijing, China). SYBR Green (Thermo Fisher Scientific Inc.)-based real-time PCR was performed with the ABI 7500 System (Applied Biosystems, Foster City, CA). Data were normalized to the internal control cyclophilin. For Western blot analysis, tissues were homogenized in lysis buffer and centrifuged. Protein concentrations in the supernatants were quantified by using the bicinchoninic acid kit. Up to $50 \mu \mathrm{g}$ of total proteins were separated by $10 \%$ SDS-polyacrylamide gel and transferred to a polyvinylidene difluoride membrane. The membrane was blocked and then incubated overnight at $4^{\circ} \mathrm{C}$ with primary anti-FABP4 antibody (D25B3, catalog number 3544) from Cell Signaling (Danvers, MA). After incubation with the secondary antibody, the membrane was treated with chemiluminescent substrate and exposed to the $\mathrm{X}$-ray film. Immunoblotting with $\beta$-actin was used as a loading control. ${ }^{13}$

\section{Serum Chemistry and Tissue Histology}

The serum levels of aspartate aminotransferase and alanine aminotransferase were measured by commercial assay kits from Stanbio (Boerne, TX). The tissue histology was evaluated by hematoxylin and eosin staining of the paraffin sections.

\section{Isolation, Culture, and $E$. coli Infection of Mouse Primary Hepatocytes}

Mouse primary hepatocytes were isolated from female C57BL/6 mice and plated onto collagen-coated 6-well plates with a density of $2 \times 10^{5}$ cells/well. After the cells were attached in the William $\mathrm{E}$ medium for 2 hours, the medium was changed to hepatocyte maintenance medium HepatoZYM-SFM from Thermo Fisher Scientific for overnight. Cells were then exposed to $0.1 \%$ of the DH5 $\alpha$ strain of Escherichia coli for 6 hours to simulate sepsis, as 
previously described. ${ }^{15}$ When applicable, $10 \mu \mathrm{mol} / \mathrm{L}$ FABP4 inhibitor BMS309403 or Ad-FABP4 was added to the medium 48 hours before the $E$. coli treatment.

\section{Statistical Analysis}

The Statistical Package for Social Sciences software version 16.0 (IBM Corporation, North Castle, NY) and GraphPad version 6.0 (GraphPad Software, Inc. La Jolla, CA) were used for statistical analyses. Results were expressed as means $\pm \mathrm{SD}$. Analysis between two individual groups was determined by the $t$-test. Survival was plotted using KaplanMeier curves, and $P$ values were calculated by log-rank (Mantel-Cox) test. $P<0.05$ was considered to be statistically significant.

\section{Results}

The Level of FABP4 in Sepsis Patients Is Positively Associated with Mortality

The Gene Expression Omnibus data set GSE54514 contains the human whole blood transcriptome of survivors and nonsurvivors of sepsis. In this data set, a positive association was found between the mRNA level of FABP4 in the blood and sepsis mortality. By both day 2 and day 3 of sepsis, the FABP4 levels in nonsurvival patients were significantly higher than in the survival group (Figure 1A). In another independent Gene Expression Omnibus data set GSE13205 that contains the skeletal muscle transcriptome in intensive care unit patients experiencing sepsis-induced multiple organ failure, the mRNA expression of FABP4 in the skeletal muscle was also found to be significantly elevated in patients with sepsis compared to their healthy control subjects (Figure 1B). These results are consistent with a recent report that the serum levels of FABP4 are positively associated with intensive care mortality. ${ }^{16}$

\section{The Hepatic Expression of FABP4 Is Specifically Induced by Sepsis}

FABP4 is abundantly expressed in the white adipose tissue and macrophages. ${ }^{5,6}$ We recently reported that the liver/hepatocytes also express FABP4. Although its basal expression in the liver was low, FABP4 was induced up to fivefold to sixfold by liver ischemia and reperfusion. ${ }^{10}$ In the same study, the mouse Fabp 4 gene was established as a hypoxia-inducible gene and a direct transcriptional target of hypoxia-inducible factor $1 \alpha .{ }^{10}$ To determine whether the hepatic expression of Fabp4 is also subjected to the regulation by sepsis, a systemic disease that involves liver injury, ${ }^{17}$ female mice were subjected to the lipopolysacharide (LPS) model of sepsis, or the cecal ligation and puncture (CLP) model of polymicrobial sepsis. ${ }^{2}$ In the LPS model, treatment of mice with LPS resulted in the induction of hepatic Fabp4 mRNA level in a time-dependent manner, with the peak induction at 16 hours
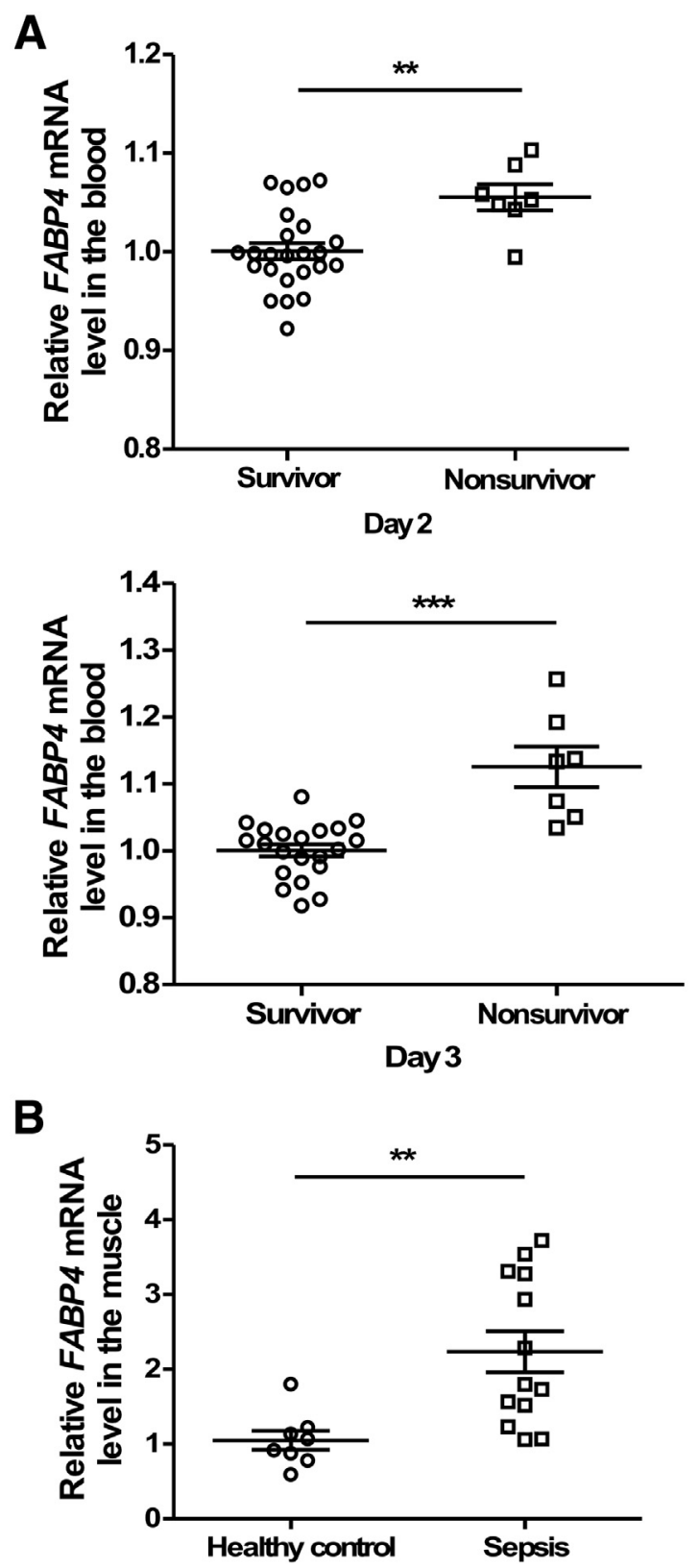

Figure 1 The level of FABP4 in sepsis patients is positively associated with mortality. A: The blood levels of FABP4 mRNA in sepsis survivors and nonsurvivors. The analysis was performed on the whole blood transcriptome data set GSE54514 from the Gene Expression Omnibus (GE0). B: The skeletal muscle levels of FABP4 mRNA in intensive care unit patients experiencing sepsis-induced multiple organ failure as compared to the healthy control subjects. The analysis was performed on the skeletal muscle transcriptome data set GSE13205 from GEO. The open circles and squares represent individual patients. ${ }^{* *} P<0.01,{ }^{* *} P<0.001$.

after the LPS injection (Figure 2A). The peak of Fabp4 induction was preceded by the induction of the inflammatory cytokine Il6 (Figure 2A). Interestingly, the induction of Fabp4 by LPS appeared to be liver specific, because the expression of Fabp4 was not induced in the gonadal white adipose tissue (WAT), but rather suppressed (Figure 2A). The expression of Il6 in WAT was induced by LPS, as expected (Figure 2A). The 
A

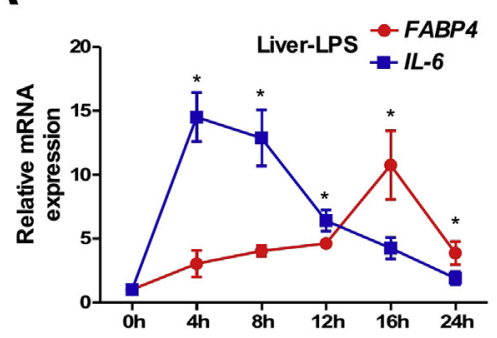

C

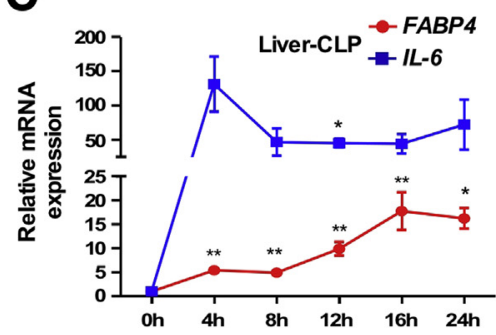

E

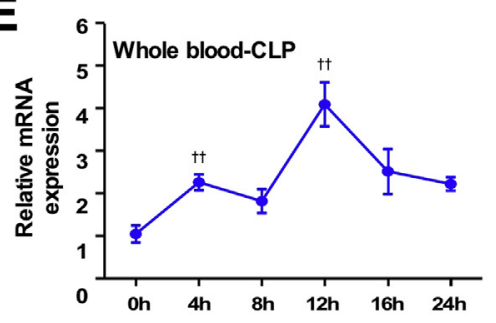

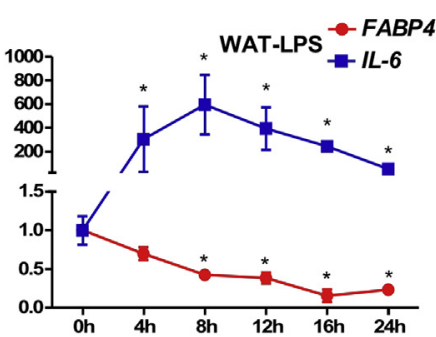
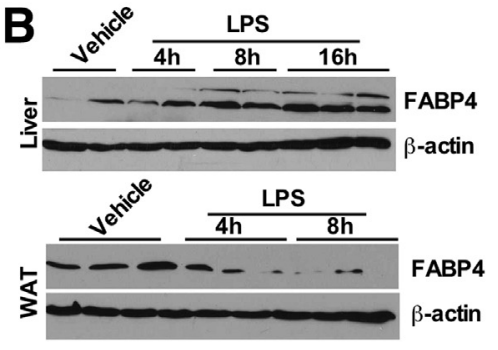

D
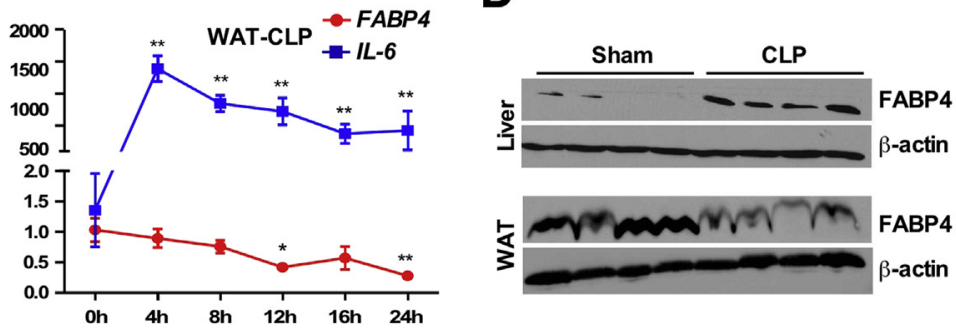

$\mathbf{F}$

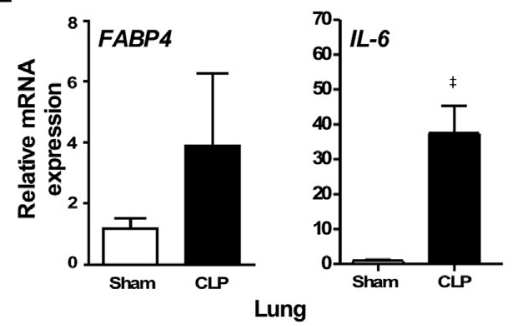

Figure 2 The hepatic expression of Fabp4 is specifically induced by sepsis. A: Mice were given a single i.p. injection of $5 \mathrm{mg} / \mathrm{kg}$ lipopolysaccharide (LPS) or vehicle before being sacrificed at the indicated time points. The mRNA expression of Fabp4 and Il- 6 in the liver and white adipose tissue (WAT) was measured by real-time PCR. The mRNA expression levels in the control groups were arbitrarily set at 1. B: The protein expression of Fabp4 in the liver and WAT of mice in A was measured by Western blotting. C: Mice were subjected to cecal ligation and puncture (CLP) or the sham surgery and then sacrificed at the indicated time points. The mRNA expression of Fabp4 and Il-6 in the liver and WAT was measured by real-time PCR. D: The protein expression of Fabp4 in the liver and WAT of mice in $\mathbf{C}$ was measured by Western blotting. E: The mRNA expression of Fabp4 in the whole blood of mice in $\mathbf{C}$ was measured by real-time PCR. F: The mRNA expression of Fabp4 and Il- 6 in the lung of mice in $\mathbf{B}$ was measured by real-time PCR. Data are expressed as means \pm SD $(\mathbf{A}, \mathbf{C}, \mathbf{E}$, and $\mathbf{F})$. $n=4$ to 5 (A and $\mathbf{C})$. ${ }^{\star} P<0.05,{ }^{* *} P<0.01$ versus the corresponding time point; ${ }^{\dagger \dagger} P<0.01$ versus 0 h; ${ }^{\ddagger} P<0.05$ versus sham.

respective induction and suppression of the protein expression of Fabp4 in the liver and WAT of LPS-treated mice were confirmed by Western blot analysis (Figure 2B). The same patterns of time-dependent Fabp4 mRNA (Figure 2C) and protein (Figure 2D) regulation in the liver and WAT were observed in mice that were subjected to CLP. The higher levels of Il6 in the liver and WAT of the CLP group (Figure 2, C and D) indicated inflammation and success of the sepsis model. CLP also induced the mRNA expression of Fabp4 in the liver of male mice (Supplemental Figure S1A), but the fold induction was not as marked as in the female mice. The expression of Fabp4 was not suppressed in the epididymal WAT of the male mice (Supplemental Figure S1B), suggesting that the sepsis-responsive regulation of Fabp4 was not completely conserved in male mice. The subsequent studies are primarily focused on the female mice.

CLP also induced the mRNA expression of Fabp4 in the whole blood (Figure 2E), consistent with the patient results (Figure 1A). However, CLP had little effect on the mRNA expression of Fabp4 in the lung, although the pulmonary expression of Il-6 was induced by CLP, as expected (Figure 2F).

\section{Kupffer Cell Depletion Does Not Attenuate the Induction of Fabp4 Sepsis}

The liver contains the Kupffer cells, which are the resident macrophages known to express FABP4. ${ }^{10}$ To determine whether Kupffer cells are required for sepsis-responsive induction of Fabp4, mice were pretreated with the Kupffer cell-depleting reagent gadolinium chloride before the LPS injection. ${ }^{18}$ The hepatic expression of Fabp4 remained induced by LPS in gadolinium chloride-treated mice (Figure 3A). Moreover, the LPS-responsive expression of Fabp4 was not significantly different between the vehicle group and the gadolinium chloride group. In contrast, the LPS-responsive induction of Il-6 was significantly attenuated in gadolinium chloride-treated mice compared to their vehicle-treated counterparts (Figure 3B). Treatment of mice with gadolinium chloride also failed to abolish the 

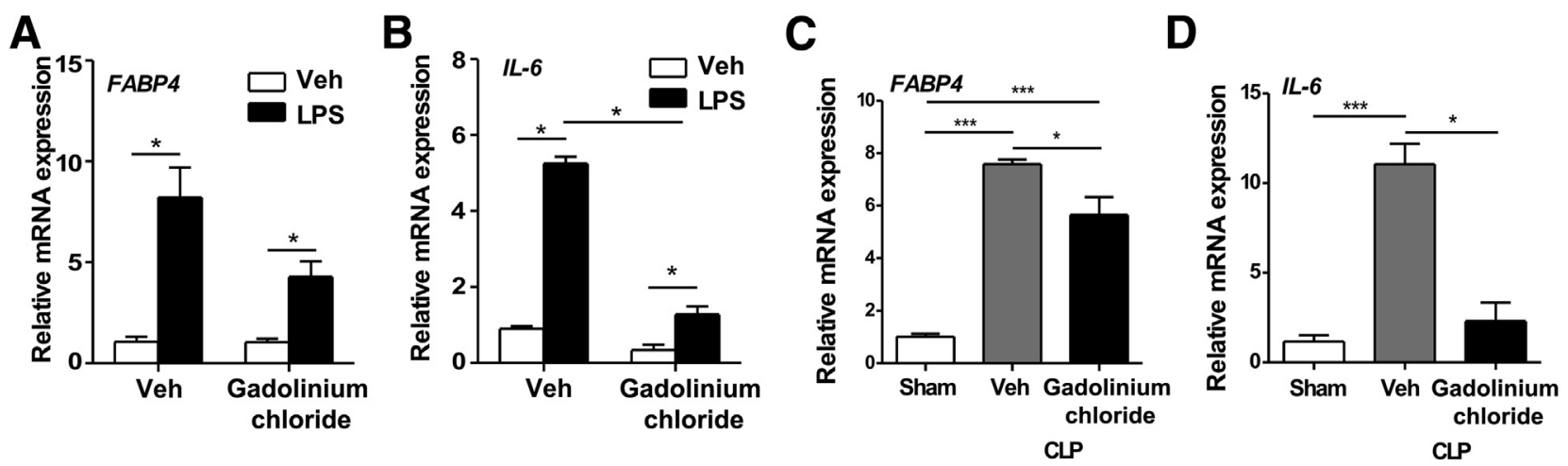

Figure 3 Kupffer cell depletion does not attenuate the induction of Fabp4 by sepsis. Mice pretreated with vehicle (Veh) or $20 \mathrm{mg} / \mathrm{kg} \mathrm{gadolinium} \mathrm{chloride}$ were subjected to a single i.p. injection of $5 \mathrm{mg} / \mathrm{kg}$ lipopolysaccharide (LPS; $\mathbf{A}$ and $\mathbf{B}$ ) or cecal ligation and puncture (CLP; C and D). Mice were sacrificed 12 hours after the LPS injection (A and $\mathbf{B}$ ) or surgery $(\mathbf{C}$ and $\mathbf{D})$ and measured for the hepatic mRNA expression of Fabp4 (A and $\mathbf{C})$ and II-6 (B and $\mathbf{D})$ by real-time PCR. The mRNA expression levels in the control groups were arbitrarily set at 1 . Data are expressed as means \pm SD $(\mathbf{A}-\mathbf{D}) .{ }^{*} P<0.05,{ }^{* * *} P<0.001$.

CLP-responsive induction of Fabp4 (Figure 3C), but the CLP-responsive induction of Il-6 was abolished in gadolinium chloride-treated mice (Figure 3D).

\section{Overexpression of Fabp4 in the Liver Sensitizes Mice to CLP}

To explore the functional relevance of sepsis-responsive induction of Fabp4, the gain-of-function liver-specific overexpression model was used to examine the effect of Fabp4 on CLP-responsive inflammation, tissue injury, and survival. In this experiment, mice were infected with adenovirus encoding the control luciferase (Ad-Luc) or mouse Fabp4 (Ad-Fabp4) 7 days before CLP. The overexpression of Fabp4 was confirmed by real-time PCR and Western blotting (Figure 4A). We found that overexpression of Fabp4 increased CLP-responsive inflammation, as supported by increased hepatic expression of Il-6 (Figure 4B) and Il-1 $\beta$ (Figure $4 \mathrm{C}$ ). The serum levels of alanine aminotransferase and aspartate aminotransferase, which indicate liver injury, were also increased in Ad-Fabp 4 infected mice (Figure 4D). Moreover, overexpression of Fabp4 significantly sensitized mice to CLP-induced lethality (Figure 4E). When the tissue histology was analyzed by hematoxylin and eosin staining, we found that in mice that were subjected to CLP, the histology of the liver (Figure 4F), heart, and kidney (data not shown) appeared to be normal and was not different between the Ad-Luc-infected mice and Ad-Fabp4-infected mice, consistent with our previous report. ${ }^{12}$ However, compared to mice infected with Ad-Luc, the Ad-Fabp4-infected mice showed profound pulmonary interstitial edema (Figure 4F), which may have contributed to the worsened survival. ${ }^{12}$

\section{Fabp4 Sensitizes Primary Hepatocytes to E. coli-Induced Sepsis Response}

The effects of CLP originate from microbial infections. Our Kupffer cell depletion experiment suggested that the
Kupffer cells did not appear to be required for the CLPresponsive induction of Fabp4 (Figure 3). To directly evaluate the effect of microbial infection on the expression of FABP4 in hepatocytes, we isolated mouse primary hepatocytes and exposed them to $E$. coli, which is an established in vitro model to simulate sepsis. ${ }^{15}$ Exposure of hepatocytes to $E$. coli was sufficient to induce the expression of Fabp4 (Figure 5A). To determine the effect of Fabp4 on E. coli-induced inflammatory response, mouse primary hepatocytes were isolated and infected with Ad-Luc or Ad-Fabp4 for 48 hours before the exposure to the DH5 $\alpha$ strain of $E$. coli. The overexpression of Fabp4 in Ad-Fabp4-infected hepatocytes was verified by both real-time PCR and Western blotting (Figure 5B). In the Ad-Fabp4-infected hepatocytes, the induction of Fabp4 at the protein level was not as dramatic as at the mRNA level, which might be because of the insufficient translation of the Fabp4 mRNA. Infection with Ad-Fabp4 increased the basal level of Il- $1 \beta$ to approximately eightfold, but not TNF- $\alpha$ (Figure 5C). The E. coli responsive expression of both Il-1 $\beta$ and Tnf- $\alpha$ was significantly higher (nearly twofold in Il- $1 \beta$ and fivefold in Tnf- $\alpha$ ) in Ad-Fabp4-infected hepatocytes than Ad-Luc infected hepatocytes (Figure 5C). In the case of Il-1 $\beta$, because of the increased basal expression of Il-1 $\beta$ in AdFabp4-infected hepatocytes, the E. coli-responsive fold induction of Il-1 $\beta$ was comparable between the Ad-Luc and Ad-FABP4 groups.

\section{Pharmacological Inhibition of Fabp4 Protects Mice from CLP}

Having shown the sensitizing effect of Fabp4 on sepsis, we next investigated whether pharmacological inhibition of Fabp4 can protect mice from CLP. In this experiment, female mice were gavaged with $25 \mathrm{mg} / \mathrm{kg}$ per day BMS309403, a pharmacological inhibitor of FABP4, ${ }^{19}$ or vehicle, for 5 consecutive days before being subjected to CLP. Treatment with BMS309403 attenuated the 

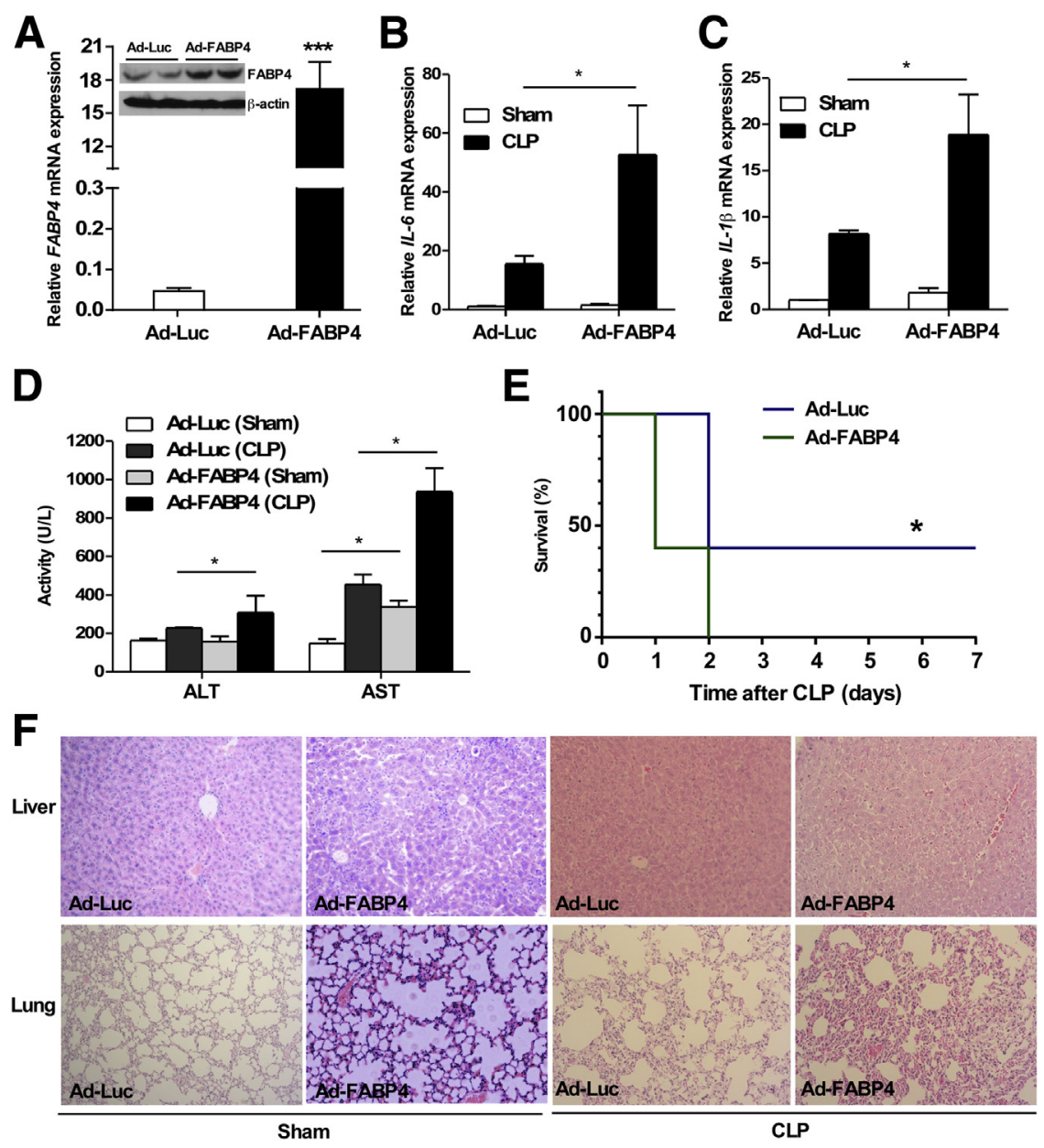

C
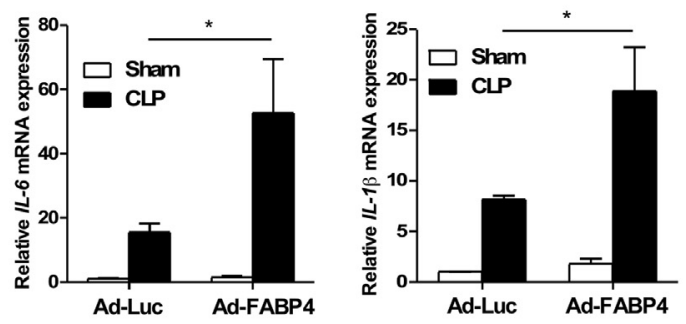

E

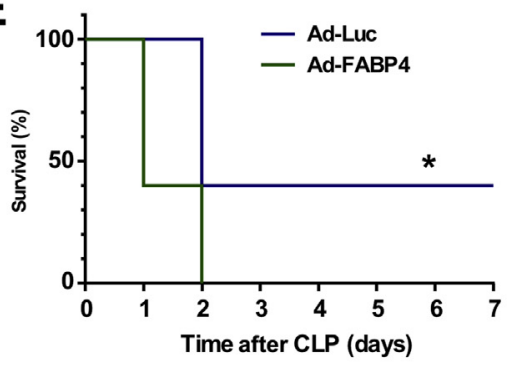

CLP

Figure 4 Overexpression of Fabp4 in the liver sensitizes mice to cecal ligation and puncture (CLP). A: The mRNA expression of hepatic Fabp4 in mice infected with Ad-Luc and Ad-Fabp 4 for 7 days was measured by real-time PCR. Inset: The confirmation of the Fabp4 protein expression by Western blotting. B and C: Mice were infected with Ad-Luc or Ad-Fabp 4 for 7 days before being subjected to CLP or the sham surgery. Mice were sacrificed 12 hours after CLP, and the hepatic expression of Il-6 (B) and Il-1 $\beta$ (C) was measured by real-time PCR. D: Mice were the same as described in $\mathbf{B}$ and $\mathbf{C}$. The serum levels of aspartate aminotransferase (AST) and alanine aminotransferase (ALT) were measured. E: Seven-day CLP survival for mice that were preinfected with Ad-Luc or Ad-Fabp4. F: The histology of the liver and lung was evaluated by hematoxylin and eosin staining. $n=5$ Ad-Luc (E); $n=5$ Ad-FABP4 (E). ${ }^{*} P<0.05,{ }^{* *} P<0.001$. Original magnification, $\times 400(\mathbf{F})$.

CLP-responsive induction of Tnf- $\alpha$, Il- 6 , and Il-1 $\beta$ in the liver (Figure 6A), as well as the elevation of the serum level of aspartate aminotransferase (Figure 6B). Moreover, treatment with BMS309403 markedly increased the animal's survival (Figure 6C). At the histological level, treatment with BMS309403 alleviated mice from CLP-induced pulmonary interstitial edema, but had little effect on liver histology (Figure 6D). In vitro, treatment of primary mouse hepatocytes with BMS309403 attenuated the
E. coli-responsive induction of proinflammatory cytokines (Figure 6E).

Pimozide is an antipsychotic drug that was recently recognized for its activity in inhibiting FABP4. ${ }^{20}$ Consistent with the results of BMS309403, pretreatment of mice with pimozide attenuated the CLP-responsive induction of proinflammatory cytokines in the liver (Supplemental Figure S2A) and interstitial edema in the lung (Supplemental Figure S2B).
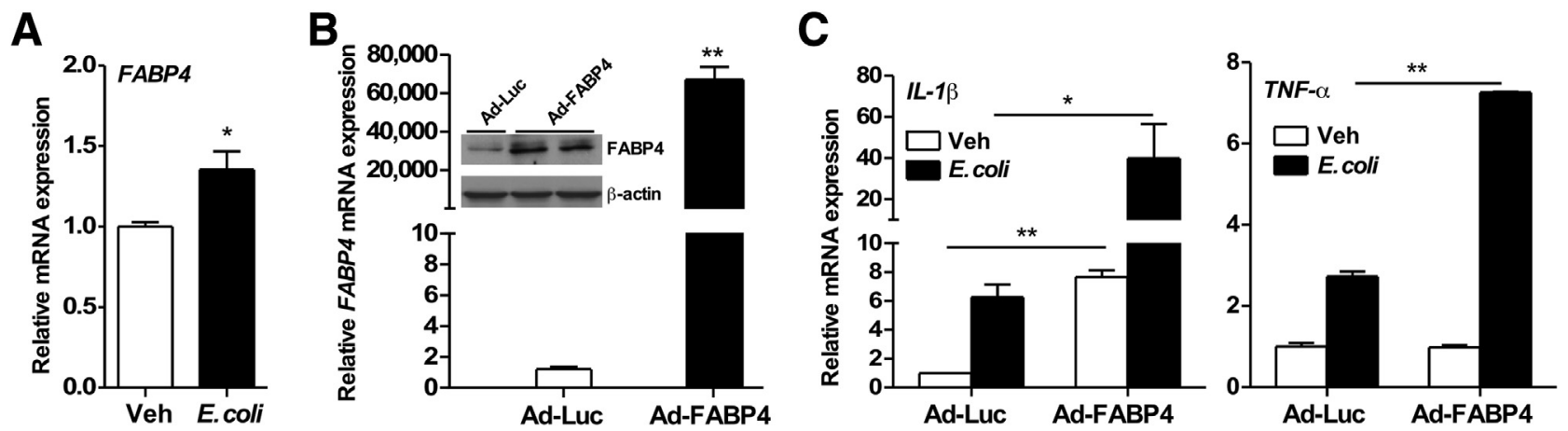

Figure 5 Fabp4 sensitizes primary hepatocytes to Escherichia coli-induced sepsis response. A: The mRNA expression of Fabp4 in mouse primary hepatocytes that were exposed to $0.1 \%$ E. coli for 6 hours. B and C: Mouse primary hepatocytes were infected with Ad-Luc or Ad-Fabp4 for 48 hours before exposure to $E$. coli $(0.1 \%)$ for 6 hours. The expression of Fabp4 (B) and Il-1 $\beta$ and Tnf- $\alpha$ (C) was measured by real-time PCR. Data are expressed as means \pm SD (A-C). ${ }^{*} P<0.05,{ }^{* *} P<0.01$. Veh, vehicle. 
A

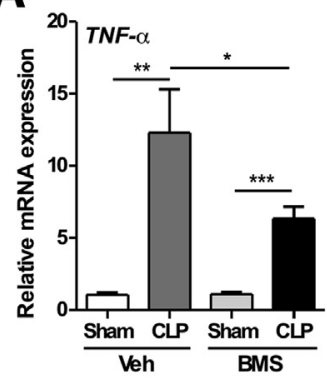

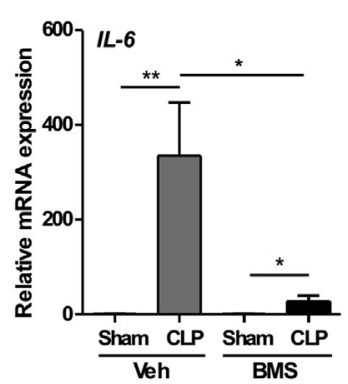

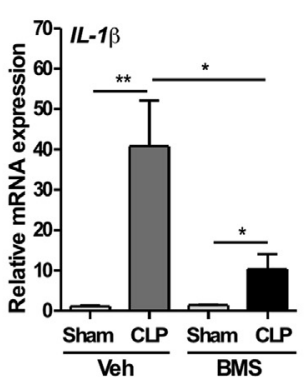

B

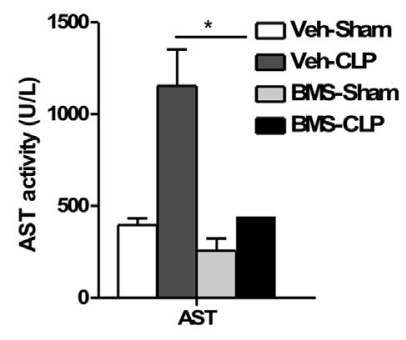

C

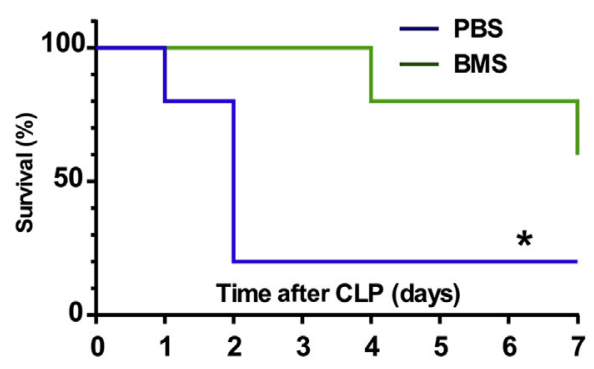

D

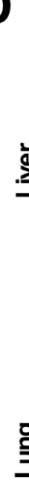

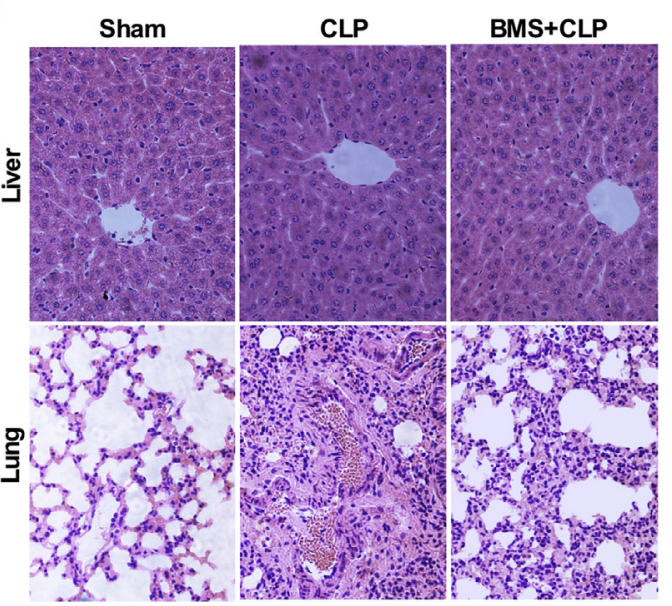

$\mathbf{E}$
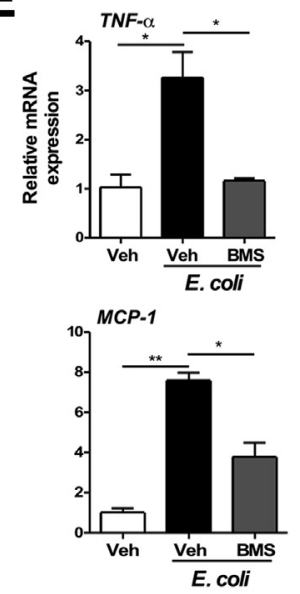

Figure 6 Pharmacological inhibition of Fabp4 protects mice from cecal ligation and puncture (CLP). Mice were subjected to oral gavage of $25 \mathrm{mg} / \mathrm{kg} \mathrm{FABP4}$ inhibitor BMS309403 (BMS) or vehicle (Veh) for 5 days before CLP. The mice were sacrificed 12 hours after the CLP surgery. A: The hepatic expression of Il-6, tumor necrosis factor (Tnf)- $\alpha$, and Il-1 $\beta$ was measured by real-time PCR. B: The serum levels of aspartate aminotransferase (AST). C: Seven-day CLP survival for mice that were pretreated with vehicle or BMS309403. D: The histology of the liver and lung was evaluated by hematoxylin and eosin staining. E: Mouse primary hepatocytes were pretreated with $10 \mu \mathrm{mol} / \mathrm{L}$ BMS309403 for 48 hours before being exposed to $0.1 \%$ Escherichia coli for 6 hours. The expression of Tnf$\alpha$ and monocyte chemoattractant protein-1 [MCP-1; alias chemokine (C-C motif) ligand 2 (CCL2)] was measured by real-time PCR. Data are expressed as means \pm SD (A, B, and $\mathbf{E}) . n=3$ for the sham groups $(\mathbf{A}) ; n=5$ for the CLP groups $(\mathbf{A}) ; \mathrm{n}=5$ PBS and BMS (C). ${ }^{*} P<0.05,{ }^{* *} P<0.01$, and ${ }^{* * *} P<0.001$. Original magnification, $\times 400$ (D).

\section{Discussion}

In this study, we showed FABP4 was induced in human sepsis patients, and an elevated level of FABP4 was associated with a worse clinical outcome. We also showed that the expression of Fabp4 was induced in animal models of sepsis. These results suggested that the induction of FABP4 plays a pathogenic role in sepsis, because ectopic expression of Fabp4 in the liver sensitized mice to CLP-induced inflammatory response and lethality, whereas pharmacological inhibition of FABP4 markedly alleviated the CLP responsive inflammation and tissue damage and improved survival.

The role of FABP4, the hepatic FABP4 in particular, in sepsis is a novel finding. Several previous reports showed that Fabp4 can sensitize mice to several inflammation-related diseases, such as obesity and carcinogenesis, and the sensitizing effect was reasoned to be because of the effect of Fabp4 on adipocytes and macrophages in the adipose tissue. ${ }^{11}$ However, the role of Fabp4 in the liver, especially in the hepatocytes, is poorly understood. Results from the current study show that although the basal expression of Fabp4 in the liver is low, its expression is highly inducible by sepsis.
Moreover, our results suggest that the hepatocytes play an essential role in sepsis-responsive induction of Fabp4 and the consequence of this induction in the pathogenesis of sepsis. This is because of the following: i) Kupffer cell depletion does not attenuate the induction of Fabp4 by sepsis, ii) exposure of primary hepatocytes to E. coli is sufficient to induce FABP4, and iii) ectopic expression of Fabp4 in the hepatocytes by adenoviral infection is sufficient to sensitize mice to sepsis. Interestingly, although hepatic overexpression of FABP4 was sufficient to sensitize mice to sepsis, the lung appeared to be more sensitive than the liver to the sepsisinduced and Fabp4-enhanced injury. However, the lung did not contribute to the sepsis-responsive induction of Fabp4 (Figure 2F).

Another interesting finding is the potential benefit of pimozide in relieving sepsis. Pimozide is an antipsychotic drug that was recently recognized for its activity in inhibiting FABP4. ${ }^{20}$ Like BMS309403, pretreatment of mice with pimozide attenuated CLP-responsive induction of inflammatory response and interstitial edema in the lung. Interestingly, pimozide was also reported to attenuate the corticosteroid resistance during pulmonary inflammation. ${ }^{21}$ 
It remains to be determined whether the inhibition of Fabp4 contributed to the therapeutic benefit of pimozide in the pulmonary inflammation. Levofloxacin is an antibiotic commonly used for sepsis patients. ${ }^{22}$ A computational screening and in vitro protein activity assays showed that levofloxacin is also a novel FABP4 inhibitor. ${ }^{23}$ It is possible that the antiseptic effect of levofloxacin may have, at least in part, accounted for its activity in inhibiting Fabp4. When FABP4 inhibitors such as BMS309403 and pimozide are used, it remains to be determined the inhibition of FABP4 in which tissue(s) are responsible for the attenuation of sepsis.

Our findings are of medical significance, because sepsis is a high mortality disease that often happens in the intensive care unit. ${ }^{24}$ As such, understanding the pathophysiology of sepsis is important for the clinical management of sepsis. In the current study, treatment of mice with FABP4 inhibitors was found to be efficient in alleviating sepsis-triggered inflammatory responses and tissue damages to the liver and lung, and improved survival. The pulmoprotective effect of FABP4 inhibitors was consistent with previous reports that the levels of FABP4 were positively correlated with the degree of pulmonary inflammation. ${ }^{25-27}$ Considering our bioinformatic finding that high levels of FABP4 were associated with poor survival in septic patients, it is tempting to speculate that FABP4 may represent an attractive target to attenuate sepsis-induced tissue injury.

The mechanism by which sepsis regulates the hepatic expression of FABP4 remains to be defined. In addition to the dispensable role of the Kupffer cells, the LPSresponsive induction of Fabp4 was found to be largely intact in $\mathrm{C} 3 \mathrm{H} / \mathrm{HeJ}$ mice that lack a functional Toll-like receptor 4 (Supplemental Figure S3), suggesting that the Toll-like receptor 4 pathway is also dispensable for this induction. The tissue-specific regulation of FABP4 by sepsis makes the mechanistic study even more challenging. A 2-kb promoter of the mouse Fabp4 gene promoter was found to be markedly suppressed by the cotransfection of p65, the major subunit of NF- $\kappa$ B (Supplemental Figure S4). This result may have explained the suppression of adipose expression of Fabp4 by sepsis, and was also consistent with a previous report that adipose-specific p65 transgenic mice had reduced Fabp 4 gene expression and improved insulin sensitivity. ${ }^{28}$

In summary, our results have uncovered a novel function of FABP4 in the pathogenesis of sepsis-induced hepatic and pulmonary injuries. We propose that FABP4 may represent a novel therapeutic target and FABP4 inhibitors may be used as therapeutic agents to manage sepsis.

\section{Acknowledgments}

We thank Dr. Karen Lam (University of Hong Kong, Hong Kong, China) for providing Ad-Fabp4 virus.
B.H., Y.L., L.G., Y.L, W.X., and M.H. are the guarantors of this work and, as such, had full access to all of the data in the study and take responsibility for the integrity of the data and the accuracy of the data analysis.

\section{Supplemental Data}

Supplemental material for this article can be found at http://dx.doi.org/10.1016/j.ajpath.2017.01.002.

\section{References}

1. Angus DC, Linde-Zwirble WT, Lidicker J, Clermont G, Carcillo J, Pinsky MR: Epidemiology of severe sepsis in the United States: analysis of incidence, outcome, and associated costs of care. Crit Care Med 2001, 29:1303-1310

2. Freitas A, Alves-Filho JC, Victoni T, Secher T, Lemos HP, Sônego F, Cunha FQ, Ryffel B: IL-17 receptor signaling is required to control polymicrobial sepsis. J Immunol 2009, 182:7846-7854

3. Nash EE, Peters BM, Fidel PL, Noverr MC: Morphology-independent virulence of Candida species during polymicrobial intra-abdominal infections with Staphylococcus aureus. Infect Immun 2016, 84: 190-198

4. Ramstead AG, Robison A, Blackwell A, Jerome M, Freedman B Lubick KJ, Hedges JF, Jutila MA: Roles of toll-like receptor 2 (TLR2), TLR4, and MyD88 during pulmonary Coxiella burnetii infection. Infect Immun 2016, 84:940-949

5. Cao H, Sekiya M, Ertunc ME, Burak MF, Mayers JR, White A, Inouye K, Rickey LM, Ercal BC, Furuhashi M, Tuncman G, Hotamisligil GS: Adipocyte lipid chaperone aP2 is a secreted adipokine regulating hepatic glucose production. Cell Metab 2013, 17: $768-778$

6. Nieman KM, Kenny HA, Penicka CV, Ladanyi A, Buell-Gutbrod R, Zillhardt MR, Romero IL, Carey MS, Mills GB, Hotamisligil GS, Yamada SD, Peter ME, Gwin K, Lengyel E: Adipocytes promote ovarian cancer metastasis and provide energy for rapid tumor growth Nat Med 2011, 17:1498-1504

7. Hotamisligil GS, Johnson RS, Distel RJ, Ellis R, Papaioannou VE, Spiegelman BM: Uncoupling of obesity from insulin resistance through a targeted mutation in $\mathrm{aP} 2$, the adipocyte fatty acid binding protein. Science 1996, 274:1377-1379

8. Furuhashi M, Tuncman G, Görgün CZ, Makowski L, Atsumi G, Vaillancourt E, Kono K, Babaev VR, Fazio S, Linton MF, Sulsky R, Robl JA, Parker RA, Hotamisligil GS: Treatment of diabetes and atherosclerosis by inhibiting fatty-acid-binding protein aP2. Nature 2007, 447:959-965

9. Hoo RL, Lee IP, Zhou M, Wong JY, Hui X, Xu A, Lam KS: Pharmacological inhibition of adipocyte fatty acid binding protein alleviates both acute liver injury and non-alcoholic steatohepatitis in mice. J Hepatol 2013, 58:358-364

10. Hu B, Guo Y, Garbacz WG, Jiang M, Xu M, Huang H, Tsung A, Billiar TR, Ramakrishnan SK, Shah YM, Lam KS, Huang M, Xie W: Fatty acid binding protein-4 (FABP4) is a hypoxia inducible gene that sensitizes mice to liver ischemia/reperfusion injury. J Hepatol 2015, 63:855-862

11. Garin-Shkolnik T, Rudich A, Hotamisligil GS, Rubinstein M: FABP4 attenuates PPAR $\gamma$ and adipogenesis and is inversely correlated with PPAR $\gamma$ in adipose tissues. Diabetes 2014, 63:900-911

12. Chai X, Guo Y, Jiang M, Hu B, Li Z, Fan J, Deng M, Billiar TR, Kucera HR, Gaikwad NW, Xu M, Lu P, Yan J, Fu H, Liu Y, Yu L, Huang M, Zeng S, Xie W: Oestrogen sulfotransferase ablation sensitizes mice to sepsis. Nat Commun 2015, 6:7979 
13. Rittirsch D, Huber-Lang MS, Flierl MA, Ward PA: Immunodesign of experimental sepsis by cecal ligation and puncture. Nat Protoc 2008, 4: $31-36$

14. Guo Y, Hu B, Huang H, Tsung A, Gaikwad NW, Xu M, Jiang M, Ren S, Fan J, Billiar TR, Huang M, Xie W: Estrogen sulfotransferase is an oxidative stress-responsive gene that gender-specifically affects liver ischemia/reperfusion injury. J Biol Chem 2015, 290:14754-14764

15. Araújo CV, Campbell C, Gonçalves-de-Albuquerque CF, Molinaro R, Cody MJ, Yost CC, Bozza PT, Zimmerman GA, Weyrich AS, CastroFaria-Neto HC, Silva AR: A PPAR- $\gamma$ agonist enhances bacterial clearance through neutrophil extracellular trap formation and improves survival in sepsis. Shock 2016, 45:393-403

16. Huang CL, Wu YW, Hsieh AR, Hung YH, Chen WJ, Yang WS: Serum adipocyte fatty acid-binding protein levels in patients with critical illness are associated with insulin resistance and predict mortality. Crit Care 2013, 17:R22

17. Yao Z, Mates JM, Cheplowitz AM, Hammer LP, Maiseyeu A, Phillips GS, Wewers MD, Rajaram MV, Robinson JM, Anderson CL, Ganesan LP: Blood-borne lipopolysaccharide is rapidly eliminated by liver sinusoidal endothelial cells via high-density lipoprotein. J Immunol 2016, 197:2390-2399

18. Wang HY, Liu XY, Han G, Wang ZY, Li XX, Jiang ZM, Jiang CM: LPS induces cardiomyocyte injury through calcium-sensing receptor. Mol Cell Biochem 2013, 379:153-159

19. Lan H, Cheng CC, Kowalski TJ, Pang L, Shan L, Chuang CC, Jackson J, Rojas-Triana A, Bober L, Liu L, Voigt J, Orth P, Yang X, Shipps GW Jr, Hedrick JA: Small-molecule inhibitors of FABP4/5 ameliorate dyslipidemia but not insulin resistance in mice with dietinduced obesity. J Lipid Res 2011, 52:646-656

20. Wang Y, Lin HQ, Law WK, Liang WC, Zhang JF, Hu JS, Ip TM, Waye MM, Wan DC: Pimozide, a novel fatty acid binding protein 4 inhibitor, promotes adipogenesis of 3T3-L1 cells by activating PPAR $\gamma$. ACS Chem Neurosci 2015, 6:211-218
21. Kabata H, Moro K, Fukunaga K, Suzuki Y, Miyata J, Masaki K, Betsuyaku T, Koyasu S, Asano K: Thymic stromal lymphopoietin induces corticosteroid resistance in natural helper cells during airway inflammation. Nat Commun 2013, 4:2675

22. Elliott TB, Bolduc DL, Ledney GD, Kiang JG, Fatanmi OO, Wise SY, Romaine PL, Newman VL, Singh VK: Combined immunomodulator and antimicrobial therapy eliminates polymicrobial sepsis and modulates cytokine production in combined injured mice. Int J Radiat Biol 2015, 91:690-702

23. Wang Y, Law WK, Hu JS, Lin HQ, Ip TM, Wan DC: Discovery of FDA-approved drugs as inhibitors of fatty acid binding protein 4 using molecular docking screening. J Chem Inf Model 2014, 54:3046-3050

24. Giuliano KK: Physiological monitoring for critically ill patients: testing a predictive model for the early detection of sepsis. Am J Crit Care 2007, 16:122-130

25. Shum BO, Mackay CR, Gorgun CZ, Frost MJ, Kumar RK, Hotamisligil GS, Rolph MS: The adipocyte fatty acid-binding protein aP2 is required in allergic airway inflammation. J Clin Invest 2006, $116: 2183-2192$

26. Ghelfi E, Yu CW, Elmasri H, Terwelp M, Lee CG, Bhandari V, Comhair SA, Erzurum SC, Hotamisligil GS, Elias JA, Cataltepe S: Fatty acid binding protein 4 regulates VEGF-induced airway angiogenesis and inflammation in a transgenic mouse model: implications for asthma. Am J Pathol 2013, 182:1425-1433

27. Zhang X, Li D, Wang H, Pang C, Wu Y, Wen F: Gender difference in plasma fatty-acid-binding protein 4 levels in patients with chronic obstructive pulmonary disease. Biosci Rep 2016, 36:e0302

28. Tang T, Zhang J, Yin J, Staszkiewicz J, Gawronska-Kozak B, Jung DY, Ko HJ, Ong H, Kim JK, Mynatt R, Martin RJ, Keenan M, Gao Z, Ye J: Uncoupling of inflammation and insulin resistance by $\mathrm{NF}-\kappa \mathrm{B}$ in transgenic mice through elevated energy expenditure. J Biol Chem 2010, 285:4637-4644 